\title{
Effect of Intrinsic Surface Roughness on the Efficiency of Intermodal Phase Matching in Silica Optical Nanofibers
}

\author{
Muhammad IM Abdul Khudus ${ }^{1,2}$, Timothy Lee ${ }^{1}$, Peter Horak ${ }^{1}$, Gilberto Brambilla ${ }^{1}$ \\ ${ }^{1}$ Optoelectronics Research Center, University of Southampton, Southampton, SO17 1BJ, United Kingdom \\ ${ }^{2}$ Photonics Research Centre, Department of Physics, Faculty of Science, University of Malaya, \\ 50603 Kuala Lumpur, Malaysia
}

\begin{abstract}
We investigate the effect of intrinsic surface roughness associated to frozen thermal oscillations from the fiber fabrication process on the efficiency of third harmonic generation via intermodal phase matching in silica nanofibers. Already a periodic wave with roughness of $0.2 \mathrm{~nm}$ reduces the efficiency by roughly $50 \%$ in a $1 \mathrm{~mm}$ optical nanofiber, with the divergence growing quadratically with distance. The surface wave period does not exhibit a large impact on the efficiency, due to averaging effects. However, both the location of the surface waves with respect to the phase matching radius as well as the surface wave amplitude have substantial effect on the efficiency, with the former presenting the possibility of transferring the power back to the pump wavelength. Simulations with a realistic superposition of random surface waves indicate that the conversion efficiency increases only for a few $\mathrm{mm}$ of propagation and reaches a maximum of less than $1 \%$.
\end{abstract}

Third order $\chi^{(3)}$ nonlinearity in centrosymmetric amorphous media can be used for efficient nonlinear optical processes, including third harmonic generation (THG) and three-photon generation (TPG), generating light at a frequency triple or one third of the pump frequency, respectively. To be efficient, these two processes require the phase matching condition to be satisfied; i.e. the effective refractive index of the pump $(\omega)$ and harmonic $(3 \omega)$ wavelengths is the same $\left(n_{\text {eff }}(\omega) \approx\right.$ $\left.n_{\text {eff }}(3 \omega)\right)$, or equivalently, the pump and harmonic wave vectors match $(\beta(3 \omega)-3 \beta(\omega) \approx 0)$. Because of material dispersion, in optical fibers phase matching can only be achieved by the transfer of power from the fundamental mode of the pump wavelength to another, typically higher order, mode of the harmonic wavelength, and is therefore known as intermodal phase matching [1]. However, in conventional telecom fibers, waveguide dispersion is considerably smaller than material dispersion, and the lack of intermodal phase matching leads to extremely low conversion efficiencies. This can be circumvented in high contrast waveguides, where the waveguide geometry can be tailored to allow for phase matching between the most efficient modes [2]. For this reason, intermodal phase matching has been proposed as means to achieve high conversion efficiency in a number of high contrast waveguides, including in optical nanofibers [3-6], microstructured fibers [7], and high numerical aperture (NA) fibers [8]. Furthermore, the effective nonlinearity in such waveguides with dimensions comparable to the wavelength is strongly enhanced by the tight modal confinement [9].

In silica nanofibers, it was initially suggested that efficiencies of more than $80 \%$ are possible for a fiber length of $50 \mathrm{~mm}$ [3]. However, the highest efficiency for a straight silica optical nanofiber achieved thus far is roughly $0.3 \%$ for a nanofiber length of $40 \mathrm{~mm}$ [5]. This might be explained by the highly susceptible nature of the conversion process to deviations from ideal phase matching resulting from subtle changes in radius due to the intrinsic roughness of silica. In this work, we investigate the effect of intrinsic, thermodynamically induced surface roughness on the efficiency of third harmonic generation (THG) in optical silica nanofibers.

The fabrication of a silica optical nanofiber is typically done by drawing heated melted glass, which is then cooled abruptly [10, 11]. These supercooled surfaces effectively freeze-in any thermally induced surface waves present during the solidification process, thereby creating an intrinsic roughness at the glass surface [12, 13]. The propagation loss in optical nanofibers as well as in microstructured optical fibers due to the surface roughness has been investigated previously by treating the roughness as a sinusoidal perturbation on the glass surface [14-16].

In this study the fiber core profile is approximated by $d=(a+\Delta a)+\Delta b c o(2 \pi z / \Lambda)$, where $a$ is the phase matching diameter, i.e. the diameter required to achieve ideal phase matching between a given pair of modes, $\Delta a$ is the average variation from the phase matching diameter, $\Delta b$ the variation amplitude, $\Lambda$ the surface wave wavelength and $z$ the distance along the fiber, as shown in Fig. 1. $\Delta a$ is used to position the surface waves at a distance from the mean position, thereby allowing the effect of the position of the surface waves on efficiency to be evaluated. The values for $\Delta a, \Delta b$ and $\Delta b+\Delta a$ are all $\leq$ $1.0 \mathrm{~nm}$, in order to be within the vicinity of the phase matching radius. 


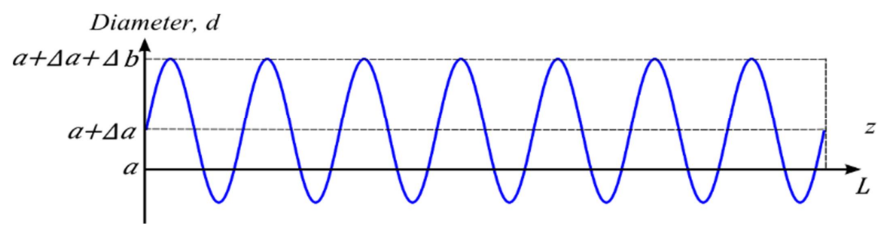

Fig. 1. Optical nanofiber surface profile. $L$ is the optical nanofiber length, $a$ is the ideal phase matching diameter, $\Delta a$ is the offset from the ideal phase matching diameter and $\Delta b$ the amplitude of the surface waves.

The phase matching conditions for THG with silica optical nanofibers is derived by evaluating the rigorous eigenvalue equation for a waveguide with a step index profile, and tailoring the index contrast and waveguide geometry [2]. In this study, we employed a pump at 1.55 $\mu \mathrm{m}$ for a third harmonic wavelength of $0.517 \mu \mathrm{m}$, with the phase matching radius being located where the effective indices $\left(n_{e f f}\right)$ of the pump in the fundamental mode and of the harmonic wavelength in a higher order mode coincide.

Once the phase matching radius is determined, the efficiency $\eta$ can be evaluated from,

$$
\begin{gathered}
\frac{\partial A_{1}}{\partial z}=-\alpha_{1} A_{1}+i n^{(2)} k_{1}\left[\left(J_{1}\left|A_{1}\right|^{2}+2 J_{2}\left|A_{3}\right|^{2}\right) A_{1}\right. \\
\left.+J_{3} A_{1}^{* 2} A_{3} e^{i \delta \beta z}\right]
\end{gathered}
$$

$$
\begin{gathered}
\frac{\partial A_{3}}{\partial z}=-\alpha_{3} A_{3}+i n^{(2)} k_{1}\left[\left(6 J_{2}\left|A_{1}\right|^{2}+3 J_{5}\left|A_{3}\right|^{2}\right) A_{3}\right. \\
\left.+J_{3}^{*} A_{1}{ }^{3} e^{-i \delta \beta z}\right]
\end{gathered}
$$

where $A_{1}$ and $A_{3}$ are the input (pump) and generated (harmonic) amplitudes, respectively; $k_{1}=2 \pi / \lambda_{1}$ the propagation constant of the pump wavelength in vacuum; $n^{(2)}$ the nonlinear refractive index of silica, $J_{n}$ the various modal vector field overlap integrals as defined in Ref. [1] and explained in Table $1 ; \delta \beta=\beta_{3}-3 \beta_{1}$ the propagation constant mismatch, or detuning, between the pump $\left(\beta_{1}\right)$ and the harmonic $\left(\beta_{3}\right)$ modes; and $\alpha_{n}$ the losses. The efficiency, $\eta$, of the THG process after a fiber length $z$ is given by $\eta=\left|A_{1}\right|^{2} /\left|A_{3}\right|^{2}$. In the following we use an adaptive step-size 4 th/5th-order Runge-Kutta method in Matlab® to solve the coupled Eqs. $(1,2)$, disregarding polarization effects.

Table 1. Interpretation of $J_{n}$ integrals

\begin{tabular}{cc}
\hline$J_{n}$ & Physical interpretation \\
\hline$J_{1}$ & Self phase modulation (SPM) of pump field \\
$J_{2}$ & Cross phase modulation (XPM) between pump \\
& and harmonic fields \\
$J_{3}$ & Energy transfer between pump and harmonic \\
$J_{5}$ & signals by THG and TPG \\
\hline
\end{tabular}

In principle, the value and changes to the value of the various $J_{n}$ should be included in the calculations of Eqs. $(1,2)$ with any change in radius. However, we found that the variation of $J_{3}$ is less than $1 \%$ in the range of radii considered, and therefore constitutes a weak effect.
Furthermore, in the limit of low pump depletion the self and cross phase modulation terms in Eqs. $(1,2)$ are mainly contributing to a constant phase shift that can be easily compensated by a small change of the nanofiber diameter a. We found numerically that for lengths of up to $10 \mathrm{~cm}$ and conversion efficiencies below 30\% the differences are negligible and thus for all simulations below we set $J_{1}=J_{2}=J_{5}=0$.

The phase matching diameter chosen was the one corresponding to the $H_{12}(3 \omega)$ mode, as initial calculations of the $J_{3}$ integral indicate that this will be the most efficient mode for THG [3-5]. The variation in diameter of the fiber is manifested mathematically as a variation in the detuning $\delta \beta$. Our calculations show that variations of $1 \mathrm{~nm}$ in radius causes changes of the order of $10^{3} \mathrm{~m}^{-1}$ in detuning, which therefore provides a limit to the variation that is employed in the fiber.

The dependence of $\eta$ on $\Lambda$ is shown in Fig. 2. For these initial calculations we limit the propagation length to 1 $\mathrm{mm}$. An optical nanofiber with a diameter $(2 a)$ of 0.7650 $\mu \mathrm{m}$ was used, with the amplitude of the surface wave, $\Delta b$, assumed to be $0.2 \mathrm{~nm}$. $\Delta a$ was set at $-0.2 \mathrm{~nm}$, in order to maximize the efficiency, as explained later. Four different surface wavelengths are considered, namely, $\Lambda=10 \mu \mathrm{m}, 1$ $\mu \mathrm{m}, 0.1 \mu \mathrm{m}$ and $0.01 \mu \mathrm{m}$, with the pump power being kept constant at $1 \mathrm{~kW}$. The wavelength of the surface wave is chosen to be similar with the values presented by Zhai et al. [14]. The optical nanofiber was assumed to be lossless.

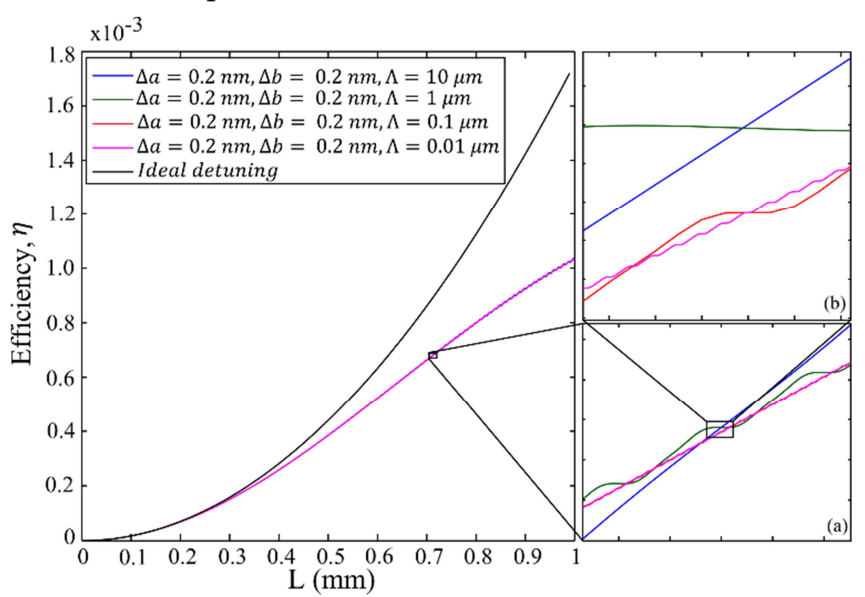

Fig. 2: Efficiency of THG in air-clad silica optical nanofiber from $1.55 \mu \mathrm{m}$ to $0.517 \mu \mathrm{m}$ for four different surface wavelengths $\Lambda$. The parameters are given above. Insets (a) and (b) show the detail of the variation in efficiency in one section of the resulting efficiency with increasing magnification to reveal the small variations that exist therein.

The efficiency of the THG process in optical nanofibers with surface waves diverges significantly from the ideal case, with the divergence being roughly $50 \%$ after $1 \mathrm{~mm}$. As the ideal phase matched case evolves initially in a quadratic manner, the divergence increases with the length of microfiber. The efficiency of the THG process in the presence of surface waves increases at a smaller rate, and exhibits an oscillatory behavior in addition to the increasing average. This is due to the fact that the surface waves are modeled as a sinusoidal variation, and therefore THG comes in and out of phase regularly. This 
also explains the behavior of the four different surface wavelengths, which do not vary enormously apart from the local variations exhibited in insets (a) and (b). The larger variation of spatially longer surface waves is because the optical nanofiber is not exactly phase matched at longer stretches than for spatially shorter surface waves. However, on average, the proportion of the phase matched lengths of the four wavelengths is similar, and therefore accounts for the small difference in efficiency.

We next investigated the effect that the position of the surface waves to the phase matching radius has on $\eta$. To this end, a $10 \mathrm{~mm}$ silica optical nanofiber was considered, with $\Delta b=0.2 \mathrm{~nm}, 2 a=0.7650 \mu \mathrm{m}$ and $\Lambda=1 \mu \mathrm{m}$. The pump power was kept constant at $1 \mathrm{~kW}$ as previously. The resulting $\eta$ is shown in Fig. 3, with the inset magnifying a small length of the optical nanofiber. The most efficient position for the optical nanofiber is where the valleys and the peaks of the surface waves coincide with the phase matching position, i.e. $\Delta a= \pm \Delta b$, because the slope of $\delta \beta$ is lower at these points and thus phase matching is achieved for longer distances, corresponding to the red and blue lines in Fig. 3. The small oscillations observed previously are also seen here, as depicted in the inset of Fig. 3, with the two most efficient positions being out of phase with each other due to the spatial difference in the two surface waves.

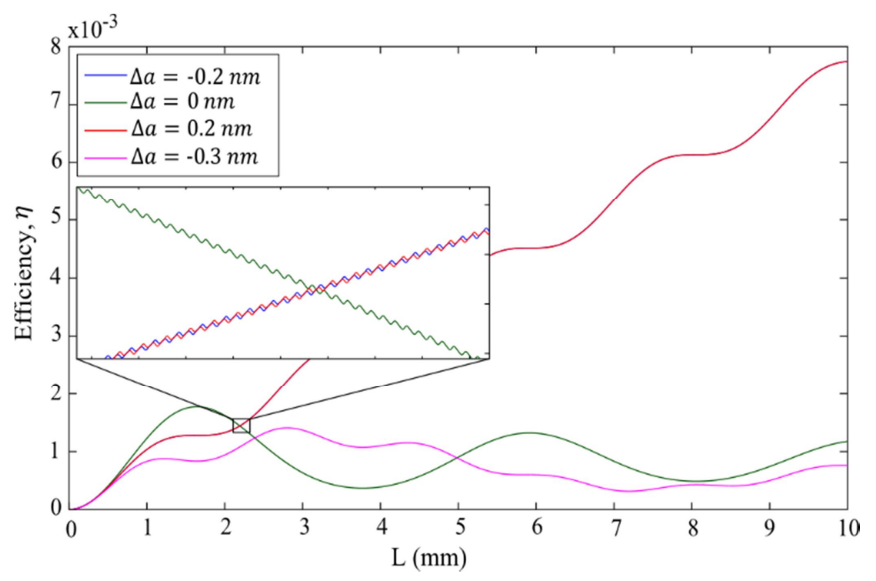

Fig. 3: Dependence of efficiency on the position of the surface waves $\Delta a$, with $\Delta b=0.2 \mathrm{~nm}$ and $\Lambda=1 \mu \mathrm{m}$. The inset shows the magnification of a small section of the resulting efficiency

The two other positions exhibit a relatively unstable behavior, with the overall efficiency first increasing over a few $\mathrm{mm}$ of the fiber before decreasing slightly. This is due to the phase dependence of the THG process, as the phase dependent $J_{3}$ coupling terms in Eq. (1) allows for the transfer of energy back to the pump wavelength. Indeed, Huang et al. [17] have shown that the same equations can be employed to describe the amplification of radiation in the mid-infrared region. An additional consequence of this term is the variation in the large 'envelope' oscillation of the four different surface waves, which is different for all four surface waves.

The influence of the surface wave amplitude on the efficiency of THG in optical nanofibers was also investigated. The same radius and peak power were employed, with $\Lambda=1 \mu \mathrm{m}$. The amplitude was varied between $-0.1 \mathrm{~nm}$ and $-0.4 \mathrm{~nm}$, with the shift in position, $\Delta a$, adjusted to provide the highest possible efficiency, $\Delta a=-\Delta b$. The resulting efficiency of the THG process is given in Fig. 4. It can be observed that increases in the amplitude of the surface waves correspond to decreases in overall efficiency, in accordance with expectations. If we magnify any part of the curve, small oscillations as seen previously are visible. However, the large 'envelope' oscillations decrease in both amplitude and wavelength with increasing surface wave wavelength. Both these observations can be explained by appealing to the fact that for smaller surface wave amplitudes, the fiber spends more time closer to the phase matching radius.

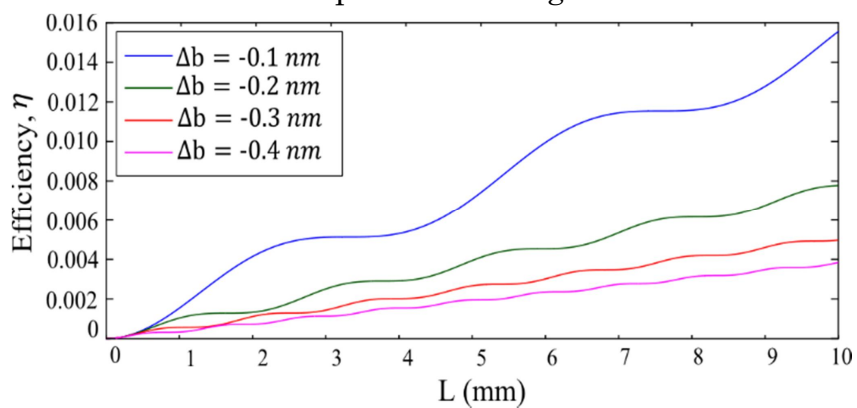

Fig. 4: Dependence of the THG efficiency on the nanofiber length for varying surface waves amplitudes $\Delta b$. In this case, $\Delta a=-\Delta b$ and $\Lambda=1 \mu \mathrm{m}$.

Finally, we simulated a $10 \mathrm{~mm}$ realistic fiber by assuming a radial profile whose spectral density, $S_{z}$, is given by [15]:

$$
S_{Z}(\kappa)=\frac{k_{B} T_{g}}{4 \pi \gamma \kappa} \operatorname{coth}\left(\frac{\kappa W}{2}\right)
$$

where $k_{B}$ is the Boltzmann's constant, $T_{g}$ is the glass solidification temperature, $\gamma$ is the surface tension and $W$ is the perimeter of the optical nanofiber. A realistic fiber is modeled by a random superposition of surface waves with a probability distribution of their amplitudes given by the spectral density (3), to produce a glass surface similar to the one measured by Roberts et al. [15].

A simulation for THG in a $10 \mathrm{~mm}$ realistic optical nanofiber for four different values of surface tension was performed. To simplify calculations, no detuning was employed in the simulation. This is justifiable as the random nature of the surface waves produces a Gaussian distribution in radial variation, which is centered roughly at the phase matching radius. The surface tension is varied in order to study the impact of the surface roughness amplitude on the efficiency.

A clear trend can be seen from Fig. 5 for all four values of surface tension, where the efficiency of the THG process increases for a short propagation distance and then stabilizes, with higher efficiencies being achieved with larger surface tensions. Mathematically, this behavior might be linked with the fact that Eq. (1) allows the transfer of energy back to the fundamental mode. It thus appears that for random surface fluctuations after an initial power transfer to the third harmonic an equilibrium is reached where an equal amount of energy 
is transferred between the two wavelengths. Higher surface tensions lead to a reduction in the radial standard deviation, increasing the overall efficiency. All of this is in contrast to the simulations both in the ideal case and when including periodic variation in radius. Note that the simulations of Fig. 5 were performed for one particular surface profile; however, we checked that the pictured results are representative for random profiles.

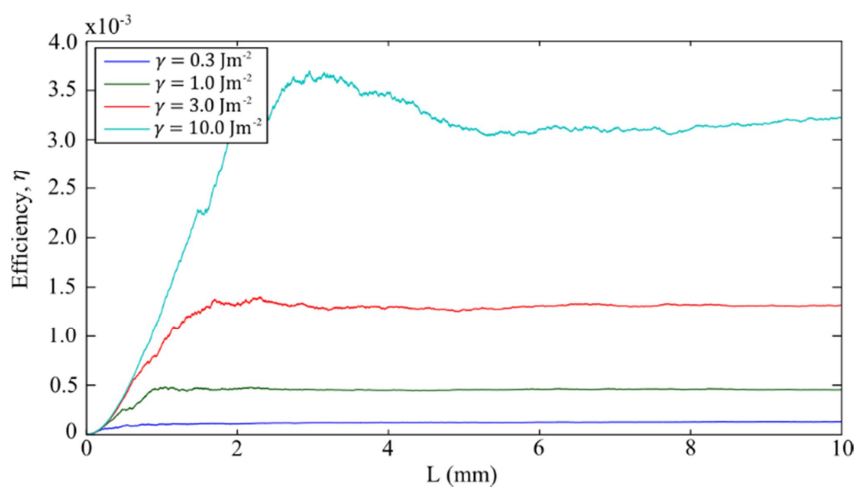

Fig. 5: Dependence of THG efficiency $\eta$ on nanofiber length L for a realistic optical nanofiber roughness profile. In this simulation, $T_{g}=1400{ }^{\circ} \mathrm{C}$ and RMS radius of approximately 383.2 $\mathrm{nm}$, with $\gamma=0.3,1.0,3.0$ and $10.0 \mathrm{Jm}^{-1}$, corresponding to a radial standard deviation of $1.18,0.67,0.36$ and $0.22 \mathrm{~nm}$.

Experimental evidence indicates that losses in optical nanofibers are of the order of $\sim 10^{-2}-10^{-3} \mathrm{~dB} / \mathrm{mm}[10$, 18]. Assuming an upper limit of roughly $0.01 \mathrm{~dB} / \mathrm{mm}$ and extrapolating from the data given by Zhai [14], a roughness of $\sim 0.3 \mathrm{~nm}$ is obtained. This indicates that the surface tension in the fiber is approximately $3.0 \mathrm{Jm}^{-1}$, slightly higher than mean value measured by Roberts et al. [15].

However, it should be noted that a higher conversion efficiency than expected from Fig. 5 for $\gamma=3.0 \mathrm{Jm}^{-1}$ was achieved by Lee et al. $(\eta \sim 0.3 \%)$ [5]. As this has little support in the literature, one explanation might be nonlinear broadening of either the fundamental or harmonic wavelengths, which allows a larger range of phase matching radii, and therefore increased efficiency. Another contributing factor is the fact that the nanofiber is not uniform, which will also increase overall efficiency. Yet another possibility is that the longitudinal distribution of surface waves might not be random and with a different period from that observed in optical fibers [16], again allowing a larger range of phase matching radii. Investigating the reasons why this behavior is observed will likely require materials with varying surface tensions and/or glass solidification temperatures.

In conclusion, we investigated the effect of surface roughness on the efficiency of intermodal phase matching in silica optical nanofibers by modelling the roughness as a sinusoidal fluctuation on the surface. To this end, THG with a pump wavelength of $1.55 \mu \mathrm{m}$ and a pump power of $1 \mathrm{~kW}$ was employed to achieve a harmonic wavelength of $0.517 \mu \mathrm{m}$. The variation in radial amplitude was kept to within $0.5 \mathrm{~nm}$ to be comparable with experimentally observed losses. The $J_{3}$ integral was assumed constant, as the variation in value is less than $1 \%$ within the $\pm 0.5 \mathrm{~nm}$ range.
We found that for a single sinusoidal surface wave its wavelength does not have a significant effect on the efficiency of THG, due to the averaging effect of the surface wave. However, the position of the surface wave with respect to the phase matching radius is important, with significant reductions in efficiency for different positions. It is also possible for the generated harmonic fields to be converted back to the pump field, which is detrimental to THG. Surface wave amplitudes have an impact on the overall efficiency, with larger surface wave amplitude corresponding to smaller efficiencies.

Simulations with realistic fibers show that the random nature of the surface wave leads to an increase in efficiency over only a few $\mathrm{mm}$ of fiber length and then stabilizes, limiting the efficiency possible through THG to less than 1\%. This is in agreement with previous experiments that also reported similar conversion efficiencies.

\section{References}

1. A.N. Naumov, and A.M. Zheltikov, Laser Phys., 12, 971 (2002).

2. T. Lee, MIM. Abdul Khudus, R. Ismaeel, C.A. Codemard, N.G.R. Broderick, and G. Brambilla, Proc. ECOC 2013, 1, (2013)

3. V. Grubsky and A. Savchenko, Opt. Express 13, 6798 (2005).

4. U. Wiedemann, K. Karapetyan, C. Dan, D. Pritzkau, W. Alt, S. Irsen, and D. Meschede, Opt. Express 18, 7693 (2010)

5. T. Lee, Y. Jung, C. A. Codemard, M. Ding, N.G.R. Broderick, and G. Brambilla, Opt. Express 20, 8503 (2012).

6. A. Coillet, and P. Grelu. Opt. Comm. 285, 3493 (2012).

7. A. Efimov, A. Taylor, F Omenetto, J. Knight, W. Wadsworth, and P. Russell. Opt. Express 11, 2567 (2003).

8. K. Bencheikh, S. Richard, G. Mélin, G. Krabshuis, F. Gooijer, and J. A. Levenson, Opt. Lett. 37, 289 (2012).

9. S.V. Afshar, M.A. Lohe, T. Lee, T.M. Monro, and N.G.R. Broderick Opt. Lett 38, 329 (2013).

10. G. Brambilla, J. Optics 12, 043001 (2010).

11. L. Tong, F. Zi, X. Guo, and J. Lou, Opt. Comm. 285, 4641 (2012).

12. J. Jackle, and K. Kawasaki, J. Phys.: Cond. Matter 7, 4351 (1995).

13. T. Seydel,, M. Tolan, B.M. Ocko, O.H. Seeck, R. Weber, E. DiMasi, and W. Press, Phy. Rev. B 65, 184207 (2002).

14. G. Zhai, and L. Tong, Opt. Express 15, 13805 (2007).

15. P.J. Roberts, F. Couny, H. Sabert, B.J. Mangan, D.P. Williams, L. Farr, M.W. Mason, A. Tomlinson, T.A. Birks, J.C. Knight, and P.St.J. Russell, Opt. Express 13, 236 (2005).

16. E.N. Fokoua, F. Poletti, and D.J. Richardson, Opt. Express 20, 20980 (2012).

17. T. Huang, X. Shao, Z. Wu, T. Lee, Y. Sun, H. Lam, J. Zhang, G. Brambilla, and S. Ping, Opt. Express 21, 28403 (2013).

18. G. Brambilla, F. Xu, and X. Feng, Elec. Lett. 42, 517 (2006) 